\title{
EFECTO DE LA SUPLEMENTACIÓN CON ENSILAJE DE NARANJA SOBRE LA CALIDAD DE LECHE CAPRINA
}

\section{EFFECT OF SUPPLEMENTATION WITH ORANGE SILAGE ON THE CAPRINE MILK QUALITY}

\author{
Dixon Fabián Flórez Delgado ${ }^{1}$, Alfonso Eugenio Capacho Mogollón ${ }^{2}$, \\ Sandra Milena Quintero Muiño ${ }^{3}$, Karen Yelitza Gamboa Vera ${ }^{4}$
}

\begin{abstract}
${ }^{1}$ Zootecnista, Magister en Sistemas Sostenibles de Producción, Docente tiempo completo ocasional. Universidad de Pamplona, Carrera 4 N. 0-14 Juan XXIII. Pamplona, Norte de Santander, Colombia, e-mail: dixon.florez@unipamplona.edu.co, (iDhttps://orcid.org/0000-0002-3915-8396; ${ }^{2} Z$ ootecnista, Magister en Calidad de la Educación Superior, Docente tiempo completo. Universidad de Pamplona, (iDhttps://orcid.org/0000-0002-0044-5566; ${ }^{3}$ Zootecnista, Especialista en Nutrición Animal Sostenible, Docente tiempo completo ocasional. Universidad de Pamplona, (iD https://orcid.org/0000-0003-0261-0542; ${ }^{4}$ Estudiante Programa de Zootecnia. Universidad de Pamplona, (DiDhttps://orcid.org/0000-0002-0745-422X
\end{abstract}

\author{
Rev. U.D.C.A Act. \& Div. Cient. 21(2):501-506, Julio-Diciembre, 2018 \\ https://doi.org/10.31910/rudca.v21.n2.2018.982
}

Artículo de acceso abierto publicado por Revista U.D.C.A Actualidad \& Divulgación Científica bajo una licencia Creative Commons CC BY-NC 4.0

\section{RESUMEN}

Uno de los principales problemas de la producción comercial de leche caprina deriva en los altos costos de suplementación, debido al uso de alimentos balanceados. El presente estudio tuvo como objetivo evaluar el efecto del suministro de ensilaje de naranja (EN) sobre la calidad de leche caprina. Se utilizó un diseño de bloques completos aleatorizados, con 48 animales de la raza Saanen, distribuidos en dos tratamientos: uno, de $250 \mathrm{~g}$ (tratamiento 1 ) y otro, de $500 \mathrm{~g}$ (tratamiento 2), de inclusión de EN y un testigo con cuatro réplicas cada una, con cuatro animales. El EN consistió en trozos pequeños del fruto, fermentados durante 30 días. Los parámetros de calidad de leche: grasa, proteína, sólidos no grasos, minerales, densidad y lactosa, se midieron en un equipo Julie C3 Scope Electric, con una periodicidad semanal, durante dos meses. Mediante comparación de medias por prueba de Tukey a los parámetros de calidad de leche, se encontró diferencia significativa $\mathrm{P}<0,05$, para grasa, en el tratamiento 1 y proteína y sólidos minerales, en el tratamiento 2 , con medias de 3,99 y $0,86 \%$, respectivamente. Para los demás parámetros, se obtuvieron valores similares. Por lo cual, se concluye que el EN, usado como suplemento alimenticio, es una alternativa nutricional, que mejora algunas características de calidad de la leche caprina.

Palabras clave: caprinos, cítricos, grasa, suplemento alimenticio, Caprinae.

\section{ABSTRACT}

One of the main problems of the commercial production of goat milk, derives in the high costs of supplementation, due to the use of balanced foods. The objective of this study was to evaluate the effect of the supply of orange silage (EN) on the quality of goat milk A randomized complete block design was used with 48 animals of the Saanen breed, distributed in two treatments, one of $250 \mathrm{~g}$ (treatment 1) and another of $500 \mathrm{~g}$ (treatment 2) of EN inclusion and a control with four replicates each with four animals. The EN consisted of small pieces of fruit fermented for 30 days. The parameters of milk quality: fat, protein, non-fat solids, minerals, density and lactose, were measured in a Julie C3 Scope Electric equipment with a weekly periodicity for two months by comparing means by means of Tukey's test to milk quality parameters, a significant difference was found $\mathrm{P}<0.05$ for fat in treatment 1 and protein and mineral solids in treatment 2 , with averages of $3.99 \%$ and $0,86 \%$ respectively. For the other parameters, similar values were obtained. Therefore, it is concluded that EN, used as a nutritional supplement, is a nutritional alternative that improves some quality characteristics of goat milk

Keywords: goats, citrus, fat, nutritional supplement, Caprinae. 


\section{INTRODUCCIÓN}

En los sistemas de producción de rumiantes, los pastos y los forrajes son la base de su alimentación, empleando alimentos concentrados a base de cereales, como principal fuente de suplementación; sin embargo, la ausencia de programas de fertilización, sistemas de riego y renovación de praderas, además de los altos costos de estos alimentos concentrados, que reducen notablemente el margen de rentabilidad para los productores pecuarios, obligan a la búsqueda de nuevas alternativas nutricionales (Bermúdez Loiza et al. 2015). Ante esta situación, los subproductos agroindustriales y residuos de cosecha constituyen una fuente importante de alimento, en la producción de carne y leche, en aquellas regiones, en donde la oferta forrajera no es suficiente (Martínez et al. 2008). El uso de subproductos generados en la agroindustria en la alimentación, especialmente en rumiantes (Pereira et al. 2008), ha permitido dar un manejo adecuado a los desechos que se generan en estos sistemas de producción, viéndose como una alternativa de solución, generando, además, muchos estudios para la búsqueda de fuentes de alimentación alternativa, en pro de reducir costos (Triana et al. 2014).

Una situación particular, se presenta en la provincia de Pamplona (Norte de Santander), en donde la naranja no se comercializa, debido a su bajo valor comercial, por lo que se están realizando estudios para su uso en nutrición de rumiantes, gracias a su composición nutricional (Velásquez et al. 2012). La naranja para alimentación animal, se puede emplear deshidratada, peletizada y molida, pero Ítavo et al. (2000) sugiere que la mejor forma de conservarla y usarla es en forma húmeda, a través del ensilaje. Los carbohidratos solubles y estructurales son sus principales aportes nutricionales, de fácil fermentación en el rumen, que favorecen la formación de ácidos propiónico y acético (Fernández, 2014).

El uso de este fruto en la alimentación animal, además de reducir los problemas de contaminación por la acumulación de residuos sobre el suelo, por la producción de malos olores, la proliferación de insectos e, incluso, filtraciones ácidas en el suelo, ha demostrado reducir la presencia de $E$. coli y Salmonella en el intestino de los rumiantes, mejorando el estado de salud de los animales, al proveer fibra, vitaminas y aceites esenciales, que actúan como un antibiótico natural. La alimentación de rumiantes con frutos de naranja y subproductos cítricos, genera tres ventajas: disminuye la dependencia y uso de cereales en la alimentación animal; se emplea como un programa de manejo y gestión de residuos agroindustriales (Grasser et al. 1995; Ajila et al. 2012) $\mathrm{y}$, finalmente, permite dar un uso a aquellos frutos que no cumplen con el estándar mínimo para su comercialización, mejorando la competitividad de este sector (Pássaro et al. 2012).
Un aspecto importante para aceptar la inclusión de pulpa de naranja en la dieta de cabras es definir si la leche y, con ello, el rendimiento quesero, uso mayoritario que se le da a la leche de cabra, sufre algún detrimento. Para ello, se han llevado a cabo diversos estudios que han ido apoyando la incorporación de este subproducto a la dieta de los animales. Entre las investigaciones realizadas destacan las de Van Horn et al. (1975), Lanza (1984), Bampidis \& Robinson (2006), que no vieron efectos adversos, como timpanismos, pérdida de condición corporal, reducción en la ganancia de peso o producción láctea en los rumiantes, tras incluir subproductos de cítricos.

Aprovechando la disponibilidad de este recurso, el objetivo del presente estudio fue evaluar el efecto de la suplementación con ensilaje de naranja sobre los parámetros de calidad de leche caprina.

\section{MATERIALES Y MÉTODOS}

Ubicación. El estudio, se realizó en la Granja Experimental Villa Marina de la Universidad de Pamplona, ubicada en la vereda Matajira del municipio de Pamplonita, Norte de Santander, Colombia. Altitud de 1.100m.s.n.m., en la parte baja y 1.800 m.s.n.m., en la parte alta; temperatura promedio de $20^{\circ} \mathrm{C}$, topografía de pendiente y precipitación de $1.400 \mathrm{~mm}$ anual.

Animales y manejo. Se emplearon 48 caprinos de la raza Saanen, con un peso promedio de $32 \mathrm{~kg}$, en fase de lactancia. Cada hembra fue identificada con un collar y se organizaron registros individuales, para realizar el seguimiento en la fase de toma de datos. Todos los animales estuvieron en pastoreo, con suplementación de sal mineralizada, de $60 \mathrm{~g}$ día. Se distribuyeron de manera homogénea en cada uno de los tratamientos: $250 \mathrm{~g}$ (tratamiento 1 ) y $500 \mathrm{~g}$ (tratamiento 2 ) de EN, además de un testigo que no recibió EN, con cuatro réplicas y cuatro hembras, por cada uno de ellos.

Elaboración del ensilaje. Para la elaboración del ensilaje de naranja (Citrus sinensis), se emplearon frutos de variedad "criolla", producidos en la Granja Experimental Villa Marina. Se tuvo como criterio de selección, los frutos de menor tamaño, debido que no tienen un buen valor comercial. Fueron picadas en trozos pequeños, para facilitar el consumo por parte de los caprinos. Se empleó el sistema de silo en bolsa calibre 7, con capacidad para 50kg, en la cual, se depositaron capas de naranja picada, seguida de una capa de harina de arroz, para reducir los niveles de humedad, hasta completar el total de la bolsa. Luego, las bolsas se llevaron a un lugar protegido del sol y de roedores para fermentación, en un periodo de 21 días (Fernández, 2014; Triana et al. 2014; Bermúdez Loiza et al. 2015). Pasado este tiempo, se verificaron las características organolépticas del ensilaje: 
color, olor y textura, así como su composición química: $\mathrm{pH}$, acidez, \% de materia seca, \% de humedad, \% de proteína, $\%$ de cenizas, \% de grasa y $\%$ de fibra, mediante análisis de laboratorio (Tabla 1).
Manejo alimenticio. La alimentación consistió en pastoreo con estrella (Cynodon plectostachyus) y braquiaria común (Brachiaria decumbens), con suplemento de $60 \mathrm{~g}$ de sal por animal día. Se aplicó un periodo de acostumbramiento del

Tabla 1. Composición nutricional del ensilaje de naranja.

\begin{tabular}{|c|c|c|}
\hline Nutriente & Contenido & Método \\
\hline $\mathrm{pH}$ & $3,63 \pm 0,04$ & Potenciometría \\
\hline Materia Seca (\%) & $13,04 \pm 1,41$ & Termo gravimétrico a $103^{\circ}$ más o menos $2^{\circ} \mathrm{C}$ \\
\hline Proteína (\%) & $8,42 \pm 0,02$ & Kjeldahl \\
\hline Cenizas (\%) & $0,56 \pm 0,03$ & Incineración directa a $600{ }^{\circ} \mathrm{C}$ \\
\hline Grasa (\%) & $1,59 \pm 0,05$ & Extracción \\
\hline Fibra (\%) & $32,88 \pm 1,1$ & Gravimétrico \\
\hline
\end{tabular}

EN de 10 días y pasado este periodo, se inició con el suministro de los tratamientos, en el momento del ordeño, dispuesto en un comedero y consumido en su totalidad, por cada uno de los animales, por lo que se sugiere que el EN fue un alimento altamente palatable (Benítez \& Poveda, 2011).

Mediciones. Pasado el periodo de acostumbramiento, los parámetros de producción diaria y calidad de la leche: grasa, proteína, sólidos no grasos, minerales, densidad y lactosa fueron medidos, empleando un equipo Julie C3 Scope Electric, con intervalos semanales, desde inicio del periodo experimental.

Análisis estadístico. Se aplicaron comparación de medias de cada tratamiento, mediante la prueba de Tukey $\mathrm{P}<0,05$ y se determinó el coeficiente de correlación de Pearson entre las variables evaluadas, con el paquete estadístico SPSS v.
20, para determinar el efecto y las diferencias entre los tratamientos y el testigo, sobre los parámetros de calidad de leche caprina.

\section{RESULTADOS Y DISCUSIÓN}

La calidad de la leche caprina presentó diferencias estadísticamente significativas en el contenido de grasa, mostrando una variación de $2,8244 \%$, en el testigo, a 5,2575\%, en el tratamiento 1, siendo diferentes entre tratamientos (Tabla 2); la densidad de la leche fue mayor para el tratamiento 1 con respecto al tratamiento 2 y testigo, con $1,0309 \mathrm{~g} / \mathrm{mL}$, $1,0299 \mathrm{~g} / \mathrm{mL}$ y $1,0299 \mathrm{~g} / \mathrm{mL}$, respectivamente.

No hubo diferencias significativas; en la concentración de lactosa, se presentaron medias de $4,4356 \%$, en el testigo, $4,9138 \%$, en el tratamiento 1 y $4,4706 \%$, en el tratamiento 2 ,

Tabla 2. Calidad de leche caprina después de suplementación con ensilado de naranja.

\begin{tabular}{|l|c|c|c|}
\hline \multirow{2}{*}{ VARIABLE } & \multicolumn{3}{|c|}{ TRATAMIENTO } \\
\cline { 2 - 4 } & TESTIGO & TRATAMIENTO 1 & TRATAMIENTO 2 \\
\hline Grasa (\%) & $2,8244 \pm 0,22^{\mathrm{a}}$ & $5,2575 \pm 0,97^{\mathrm{b}}$ & $4,5556 \pm 0,43^{\mathrm{a}}$ \\
\hline Densidad & $1,0299 \pm 0,34^{\mathrm{a}}$ & $1,0309 \pm 0,31^{\mathrm{a}} \mathrm{a}$ & $1,0299 \pm 0,52^{\mathrm{a}}$ \\
\hline Lactosa (\%) & $4,4356 \pm 0,07^{\mathrm{a}}$ & $4,9138 \pm 0,97^{\mathrm{a}}$ & $4,4706 \pm 0,24^{\mathrm{a}}$ \\
\hline SNG (\%) & $8,1769 \pm 0,10^{\mathrm{a}}$ & $9,0481 \pm 1,76^{\mathrm{a}}$ & $8,1856 \pm 0,18^{\mathrm{a}}$ \\
\hline Proteína (\%) & $3,0575 \pm 0,04^{\mathrm{a}}$ & $3,3806 \pm 0,65^{\mathrm{a}}$ & $3,9975 \pm 0,41^{\mathrm{b}}$ \\
\hline Solidos Minerales (\%) & $, 6250 \pm 0,01^{\mathrm{a}}$ & $, 6819 \pm 0,12^{\mathrm{b}}$ & $, 8669 \pm 0,04^{\mathrm{c}}$ \\
\hline
\end{tabular}

a, b, c $=$ Medias con distinta letra son diferentes estadísticamente $(P<0,05)$. 
mientras que, en los sólidos no grasos, los valores fueron de $8,1769 \%, 9,0481 \%, 8,1856 \%$, todos en el mismo grupo, lo que indica que no se presentaron diferencias.

El contenido de proteína fue similar en el testigo y el tratamiento 1 , con 3,0575 y $3,3806 \%$, respectivamente, siendo diferentes con el tratamiento 2, con 3,9975\% (Tabla2). En los sólidos minerales fue mayor en el tratamiento 2, presentando diferencia estadística con el testigo y tratamiento 1 , $0,6250 \%$ y $0,6819 \%$, respectivamente (Tabla 2 ).

El aumento del contenido en estos parámetros, se debe a la activación de la acetil-Coa carboxilasa, debido al citrato citoplasmático presente en la glándula mamaria, para el proceso de lipogénesis (Gumaa et al. 1970). Por su parte, Volanis et al. (2014) y Belibasakis \& Tsirgogianni (1996) explican que el EN promueve condiciones favorables para la actividad microbiana del rumen, debido a la fibra de alta digestión que ofrece, aumentando, así, la producción de ácido acético, como precursor de ácidos grasos, conllevando a la síntesis de grasa en la leche (Wing, 1982; Belibasakis \& Tsirgogianni, 1996), fermentación de pectinas (Strobel \& Russell, 1986) y cambio moderados en el pH del rumen (Villareal et al. 2006; Piquer et al. 2009), evitando la presencia de acidosis (Wing,1982). Estas ventajas hacen de este suplemento una buena fuente de energía alternativa a bajo costo, como sustituto de los alimentos balanceados comerciales.

Otros estudios similares, realizados por Belibasakis \& Tsirgogianni (1996), muestran un aumento de $4,12 \%$ de grasa en la leche, siendo inferiores a los reportados por el presente estudio, 4,5 a 5,2\%. Finalmente, Tafoya (2011) reporta resultados similares a los de esta investigación, con un rango que osciló entre 3,15 y 3,7\%, de grasa en la leche, al evaluar porcentaje de inclusión de EN, del $42 \%$ y $86 \%$, de sustitución de sorgo en la dieta de los caprinos. Santiago (2007) señala que un porcentaje del 3,4\% de proteína en la leche es un indicativo de calidad en este producto.

El nivel de correlación entre las variables asociadas a la calidad de la leche (Tabla 3) evidenció que existe una relación lineal fuerte entre las variables densidad de la leche y contenido de lactosa $\left(, 940^{* *}\right)$, lo que indica que, a medida que la densidad aumentó, el contenido de lactosa fue mayor; asimismo, existe una relación lineal fuerte entre la densidad y los sólidos no grasos $\left(, 955^{* *}\right)$, pues a medida que el contenido de estos últimos se incrementaron, la densidad se comportó de la misma forma. Por otro lado, no se observó relación lineal entre las variables densidad y grasa $\left(, 388^{* *}\right)$, pues el valor de $p$ es relativamente cercano a 0 , lo que indica que el contenido de grasa no influencia de forma lineal o directa sobre la densidad y que no se requiere un nivel mínimo de grasa para que exista una densidad determinada.

Los resultados del presente estudio indican que el ensilaje de naranja podría ser una alternativa nutricional viable para la alimentación de caprinos productores de leche, sin efectos adversos para los animales. Los frutos, cuyas características no permitan su comercialización o aquellos frutos que por condiciones de precio no sean viables para llevar al mercado por parte de los productores, pueden ser empleados en la alimentación animal, siendo una opción de manejo adecuado de residuos agrícolas. La incorporación de EN en la dieta, con $250 \mathrm{~g}$ en la alimentación de caprinos, aumentó significativamente el contenido de grasa, mientras que la inclusión de $500 \mathrm{~g}$ favoreció el contenido de proteína y DE solidos minerales, sin afectar el contenido de los demás parámetros de calidad, siendo muy favorable al productor, ya que mejoraría sustancialmente los ingresos por comercialización de leche de mejor calidad.

Tabla 3. Coeficiente de correlación de Pearson entre las variables de calidad de la leche.

\begin{tabular}{|c|c|c|c|c|c|c|}
\hline & Grasa & Densidad & Lactosa & SNG & Proteína & Sol. Min \\
\hline Grasa & ---- &, $388^{* *}$ &, $562^{* *}$ &, $559^{* * *}$ &, $576^{* * *}$ &, $536^{* * *}$ \\
\hline Densidad & & ---- &, $940^{* *}$ &, $955^{* * *}$ &, $615^{* * *}$ &, $482^{* *}$ \\
\hline Lactosa & & & ---- &, $981^{* * *}$ &, $618^{* * *}$ &, $414^{* * *}$ \\
\hline SNG & & & & ---- &, $552^{* * *}$ &, $405^{* * *}$ \\
\hline Proteína & & & & & ---- &, $894^{* * *}$ \\
\hline Sol. Min & & & & & & ---- \\
\hline
\end{tabular}

* La correlación es significativa en el nivel 0,01.

Nota: SNG: Solidos No Grasos, Sol. Min: Solidos Minerales. 
Conflictos de intereses: El manuscrito fue preparado y revisado con la participación de todos los autores, quienes declaramos que no existe conflicto de intereses que ponga en riesgo la validez de los resultados presentados.

\section{REFERENCIAS}

1. AJILA, C.M.; BRAR, S.K.; VERMA, M.; PRASADA RAO, U.J.S. 2012. Sustainable solutions for agro processing waste management: An overview. In: Malik, A.; Grohmann, E. (Eds). Environmental Protection Strategies for Sustainable Development. Netherlands: Springer. p.65 109.

2. BAMPIDIS, V.A.; ROBINSON, P.H. 2006. Citrus by products as a ruminant feed: a review. Anim. Feed Sci. Technol. 128:175-217. https://doi.org/10.1016/j. anifeedsci.2005.12.002

3. BELIBASAKIS, N.; TSIRGOGIANNI, D. 1996. Effects of dried citrus pulp on milk yield, milk composition and blood components of dairy cows. Anim. Feed Sci. Technol. (Holanda). 60(1-2):87-92. https://doi. org/10.1016/0377-8401(95)00927-2

4. BENIITEZ, S.; POVEDA, C. 2011. Evaluación nutricional de ensilajes con diferentes niveles de inclusión de cáscara de naranja (Citrus sinensis) y digestibilidad in vivo como alternativa energética para alimentación de cerdos. Rev. Col. Ciencia Animal. 4(1):20-28.

5. BERMÚDEZ-LOAIZA, J.A.; MELO-CAMACHO, E.P.; ESTRADA-ÁLVAREZ, J. 2015. Evaluación de ensilaje de naranja entera (Citrus sinensis) como alternativa de suplementación en bovinos. Rev. Veterinaria y Zootecnia. 9(2):38-53. https://doi.org/10.17151/ vetzo.2015.9.2.4

6. FERNÁNDEZ, A. 2014. Transformación de subproductos y residuos de agroindustria de cultivos templados, subtropicales y tropicales en carne y leche bovina. Instituto Nacional de Tecnología Agropecuaria. Centro Regional Buenos Aires Sur Estación Experimental Agropecuaria Bordenave EEA Bordenave. $1^{\mathrm{a}}$ edición. 200p.

7. GRASSER, L.A.; FADEL, J.G.; GARNETT, I.; DEPETERS, E.J. 1995. Quantity and economic importance of nine selected by-products used in California dairy rations. J. Dairy Science. 78(4):962-971. https://doi. org/10.3168/jds.S0022-0302(95)76711-X

8. GUMAA, K.; GTEENBAUM, A.; MCLEAN, P. 1970. The control of pathways of carbohydrate metabolism in mammary gland. In: I.R. Falconer (Editor), Lactation. Butterworths, London. p.197-238.

9. ÍTAVO, L.C.; DOS SANTOS, G.T.; JOBIM, C.C.; VOLTOLINI, T.V.; FERREIRA, C.C. 2000. Substituicão da silagem de milho pela silagem do bagaco de laranja na alimentacão de vacas leiteiras. Consumo, producão e qualidade do leite. Rev. Brasileira de Zootecnia. 29:1498-1503.

10. LANZA, A. 1984. Dried citrus pulp in animal feeding. In: Hollo, J. (Ed.), Proceedings of the International Symposium on Food Industries and the Environment. Budapest, Hungary. Elsevier Pulishers, New York, NY, USA. p.189-198.

11. MARTIINEZ, J.; CHONGO, B.; JORDÁN, H.;HERNÁNDEZ, N.; FONTES, D.; LEZCANO, Y.; CUBILLAS, N. 2008. Características nutritivas de los hollejos húmedos de naranja (Citrus sinensis cv. Valencia) mantenidos en estibas. Técnica Pecuaria en México. 46(2):183-193.

12. PÁSSARO, C.; NAVARRO, P.; SALVADOR, A. 2012. Poscosecha. In: Garcés, L.F. (Ed.). Cítricos: cultivo, cosecha e industrialización. Caldas: Corporación Universitaria Lasallista. p.223-284.

13. PEREIRA, M.; DE AZAMBUJA, E.; MIZUBUT, I.; DA ROCHA, M.A.; KURAOKA, J.; NAKAGHI, E. 2008. Consumo de nutrientes e desempenho de cordeiros em confinamento alimentados com dietas com polpa cítrica úmida prensada em substituição à silagem de milho. Rev. Brasileira Zootecnia. 37:134-139. http:// dx.doi.org/10.1590/S1516-35982008000100020

14. PIQUER, O.; RÓDENAS, L.; CASADO, C.; BLAS, E.; PASCUAL, J.J. 2009. Whole citrus fruits as an alternative to wheat grain or citrus pulp in sheep diet: Effect on the evolution of ruminal parameters. Small Ruminant Research. 83(1):14-21. https://doi. org/10.1016/j.smallrumres.2008.11.009

15. SANTIAGO, M. 2007. Manual de normas de control de calidad de leche cruda. Ciudad de México: Secretaría de Desarrollo Social (SEDESOL).

16. STROBEL, H.; RUSSELL, J. 1986. Effect of $\mathrm{pH}$ and energy spilling on bacterial protein synthesis by carbohydrate-limited cultures of mixed rumen bacteria. J. Dairy Science. 69(11):2941-2947. https://doi. org/10.3168/jds.S0022-0302(86)80750-0

17. TAFOYA, M. 2011. Substitución parcial del grano de sorgo por cáscara de naranja deshidratada y factores 
de variación en la producción de cabras. Universidad Autónoma de San Luis Potosí. México. 60p.

18. TRIANA, E.; CAMPO, Y.; LIZCANO, H. 2014. Evaluación de ensilaje a partir de dos subproductos agroindustriales (cascara de naranja y plátano de rechazo) para alimentación de ganado bovino. Rev. Alimentos Hoy. 22:31-45.

19. VAN HORN, H.H.; MARSHALL, S.P.; WILCOX, C.J.; RANDEL, P.F.; WING, J.M. 1975. Complete rations for dairy cattle. III. Evaluation of protein percent and quality, and citrus pulp-corn substitutions. J. Dairy Sci. 58:1101-1108. https://doi.org/10.3168/jds. S0022-0302(75)84688-1

20. VELÁSQUEZ, R.; ESQUIVEL, H.; MONTERO, L.; KU, J. 2012. Engorda de corderos Pelibuey con ensilaje de pulpa de naranja Citrus sinensis L. en jaulas elevadas. Rev. Col. Ciencia Animal. 5(1):67-31.
21. VILLARREAL, M.; COCHRAN, R.C.; ROJAS-BOURRILLÓN, A.; MURILLO, O.; MUÑOZ, H., POORE, M. 2006. Effect of supplementation with pelleted citrus pulp on digestibility and intake in beef cattle fed a tropical grass-based diet (Cynodon nlemfuensis). Animal Feed Science and Technology. 125:163-173. https://doi.org/10.1016/j.anifeedsci.2005.05.020

22. VOLANIS, M.; ZOIOPOULOS, P.; TZERAKIS, K. 2014. Effects of feeding ensiled sliced oranges to lactating dairy sheep. Small Rumin. Res. 53:15-21. https://doi. org/10.1016/j.smallrumres.2003.07.011

23. WING, J. 1982. Citrus feed stuffs for dairy cattle. Gainesville: University of Florida. 16p.

Recibido: Febrero 20 de 2018

Aceptado: Octubre 5 de 2018

Cómo citar

Flórez Delgado, D.F.; Capacho Mogollón, A.E.; Quintero Muiño, S.M.; Gamboa Vera, K.Y. 2018. Efecto de la suplementación con ensilaje de naranja sobre la calidad de leche caprina. Rev. U.D.C.A Act. \& Div. Cient. 21(2):501-506. https://doi. org/10.31910/rudca.v21.n2.2018.982 